\title{
Participatory Rural Appraisals (PRA) for Watershed Management (A Case Study of Chapramari Watershed)
}

\author{
Mrinal Kanti Roy*
}

West Bengal Forest Service, Jalpaiguri Soil Conservation Division and Project Implementing Agency, Chapramari Watershed (IWMP-5/11-12/batch-III, District-Jalpaiguri), India

*Corresponding author

\section{A B S T R A C T}

Keywords

Watershed, PMKSY, IWMP PIA, PRA, PFMS, GIS, MIS

Article Info

Accepted:

12 December 2017

Available Online:

10 January 2018
The PMKSY (Pradhan Mantri Krishi Senchaya Yojona) is a central Government scheme started with an object of wholistic development of the community resides in a particular watershed. This case study is mean to try to focus upon the Participatory management of watershed and its operation and achievement and empowering the villagers for their own development.

\section{Introduction}

Watershed is a natural unit draining runoff water to a common point (i.e. drainage basin) demarcation based on ridge and gully lines. It can also be said that it is a natural hydrologic entities that cover a specific area expanse of land surface from which rainfall runoff drains to a defined drain/cannel/stream or river at a particular point. The size of the watershed can be selected depending upon the possibilities of development within 3-5 years. The project of Chapramari Watershed is being implemented through the PIA (Project Implementing Agency) cum Divisional Forest Officer, Jalpaiguri Soil Conservation Division,
Directorate of Forests, Government of West Bengal under the part of Integrated Watershed Management Project (IWMP) now renamed as Pradhan Mantri Krishi Senchay Yojona (PMKSY) under Department of Land Resources, Government of India.

It is a project of the watershed community with Government's participation. The watershed approach is more rational because land and water resources have optimum interactions and synergetic effect when developed in watershed basis for total development of all the community members in the delineated micro watershed. 


\section{Materials and Methods}

\section{Base line data}

The Chapramari watershed project, IWMP-5 / 2011-12 covering the Upper Tendu Forest (Jl. no 1M) Mouza of Nagrakata Block, Jalpaiguri District. It is located at $26^{\circ} 44^{\prime} 17.84 " N$ to $26^{\circ} 57^{\prime} 10.62^{\prime \prime} \mathrm{N}$ Latitude and $88^{\circ} 49^{\prime} 44.84^{\prime \prime} \mathrm{E}$ to $88^{\circ} 52^{\prime} 46.88^{\prime \prime E l o n g i t u d e . ~ T h e ~ w o r l d ~ f a m o u s ~}$ Chapramari Wildlife Sanctuary is a part of the watershed. The geographical area of the Watershed is $8984.13 \mathrm{Ha}$ and the Treatable Area is $4300 \mathrm{Ha}$. It comprises 04 no. of micro watersheds namely a. Sipchu Micro Watershed b. Panjhora Micro watershed c. New Khunia Micro watershed d. North Indong Micro watershed. All of them are recognised as Forest Village settlement. It comprise of $65.74 \%$ of marginal farmers and $34.25 \%$ are small farmers. The main livelihood support is Forestry (daily labours) and agriculture. The area is scarce of assured irrigation, so the kharif paddy is the only source of agricultural output. The objective of undertaking the project in this area is to support livelihood by way of enhancing production and productivity with both way of extensive and intensive cultivation. There is ample scope of alternative source of income by rearing cattle, goat etc. in a scientific approach. These can be done when maximum rain water is harvested in situ for using in production purpose keeping in mind regeneration of degraded natural resources like soil, water and plantation. The asset less persons seeks their livelihood from the natural plantation/forest. The improvement of cattle breed by artificial insemination, immunization particularly for foot and mouth disease of cattle, de-worming among others can pave the way of enhanced additional income of the community. On the other hand integrated farming system approach can be well developed for better use of farm waste recycling. While considering technological intervention, the ITK and Low Cost
Technology would be adopted. The literacy percentages among SC/ST and Minorities are far lower than the others. So the tools to educate the people of these communities will be considered for better adoption. The constitution of Watershed Committee has been done with more than $50 \%$ of the members is from SHGs/ UGs comprising SC/ST/women and asset less persons.

\section{Surveying}

To implement watershed area program systematically the survey has been conducted for knowing the potentiality of the village. The survey will serve as a bench mark against which the results of project could be compared at the end of the implementation. It would also be helpful in guiding watershed program to plan its goal in identifiable terms for future reference. PRA techniques and Focused Group Discussion (FGD) were conducted with the residents of the micro watershed members and beneficiaries for building confidence for participation during project planning.

\section{Cluster approach}

This envisages a broader vision of Geohydrological unit which involves treating the cluster of 4 micro watersheds namely Sipchu, Panijhora, New Khunia and North Indong of Chapramari Watershed. This watershed is not in contagious to with other watershed projects to be undertaken in the area.

\section{Collection of primary data}

Though the project was sanctioned by the September, 2011 but the preparatory phase started in 2013. Initially, a meeting was arranged with officials of concerned departments and technical experts located at the District Headquaters at Jalpaiguri and also near the micro- watersheds. During this meeting, preliminary details of the proposed 
project including location of villages and criteria of selection and PPR were discussed.

In order to have first-hand information, a joint visit in the project area was made along with PIA members. In this survey, physical location of the watershed, important villages, drainage system, main land use and other problems related to the area were assessed. The Pradhans of the Gram Panchyet and local people were involved in the discussions and a note of the local needs and scope of watershed works was taken up.

High resolution maps depicting the physical condition of the area on the 1:50000 scales were procured from NRSC, GOI and SRTM data were incorporated into the maps which included the physical parameters which are present in the area.

\section{Collection of secondary data}

The data with regard to Demographic, socioeconomic, infrastructure, land use, primary and secondary occupation, major crops grown and the yield level of vegetable crops, marketing facilities, fodder production, agroforestry crops, livestock and milk production, status of self-help groups, previous watershed schemes and works undertaken under MGNREGA etc. was gathered with the help of a specially designed questionnaires by social development associates.

Additional information was gathered by group and individual discussions with women groups, landless and other poor sections of the society. The issues concerning water availability, use of common property resources, fuel and fodder availability, wage employment opportunity, livelihood opportunities, development of microenterprise and other major concerns were discussed, debated and recorded.

\section{Participatory Rural Appraisal (PRA)}

The due process of participatory Appraisal was followed in which village committees were sensitized about project activities. An appraisal of land resources, water resources, forest and pasture land resources, common property resources, production system and livestock resources was carried out by collecting data from primary sources. Group meeting were organized at common places and problem and possible solution were debated, discussed and efforts were made to reach agreement on activities required under the projects. This was followed by transit walks across the entire area of the village and spots indicated by the community. Focused Group Discussion (FGD) was carried out in which Technical possibilities were discussed and measurements were recorded for jointly agreed activities. Similarly, discussions were held about entry point activities and the progress of work was ascertained. The remaining works, which were under progress was also discussed with the villagers and the officials from the PIA. The works were finalized keeping in view the availability of funds in the project.

Through discussion were held on production activities and new innovative techniques of improving crop, fruit and milk production. The women groups were sensitized about income generating activities and skill improvement by various types of trainings. The department field staff initiated the process of participation at the planning stage. The department officials simultaneously stated the process of forming watershed committees for each village. The roles and responsibilities of all stake holders as per guidelines, the mechanism of fund flows, cost sharing arrangement in different components, and operational mechanism of the projects was thoroughly discussed with the community and to the Watershed Committees (WC) in detail. 


\section{Participatory net planning}

The action plan was formulated based on the PRA, Geo-hydrological condition, Drainage pattern, Soil class, Soil erosion, forest and agriculture land. The project proposals were deliberated in the Gram Sabha meetings which were approved with required amendments.

Based on the experience of the resource persons in the area and catchment area characteristics, structures like Water Conveyance System, Dry Stone Check Dams etc. were recommended to conserve and store water used for life saving additional irrigation potential in the rain fed area and to avoid degradation of the land.

\section{Community participants in social mapping}

The village communities were apprised about project activities. Group meetings were organized at common places, problems and possible solutions were debated, discussed and efforts were made to reach agreement on activities required under the project. Social mapping involving local community was prepared.

Infrastructure services and other village resources such as ponds, wells, agriculture land etc. were mapped.

\section{Transact walk}

Reconnaissance survey was carried out through transect walk in order to identify the needs, treatments required and worksites. The sites were marked on the maps and different treatment measures required were recommended.

\section{Focus group discussion}

Focus group discussions (FGD) were conducted in order to obtain communities' approval on various identified needs. It was helpful in complementing the assessment emerged from PRA and to derive the opinion of the communities on various issues.

\section{Use of GIS technology in planning}

Use of high scientific tools has been promoted at various stages of watershed development planning. Geographical Information System (GIS) has been used in planning. Various layer maps were created likes Base map, Present Land Use, Geo-hydrological, Micro Watershed, Drainage, Contours, Soil Classification, Land Capability Classification, Ground Water, Proposed and existing Activities or works.

\section{Prioritization}

With the assistance of Geographical Information System (GIS), various layers were created like Topography (slope), Drainage and contour, Slope, soil and Land Capability classes. All these parameters were given weight age as per the guidelines issued by Govt. of India. This has helped in prioritization of various watershed areas.

\section{Planning}

Based on the land use and hydrology maps in addition to social maps (PRA) prepared by the participants, analysis was carried out for the planning in micro- watersheds. The action plan was formulated based on Geohydrological condition, Drainage pattern, Soil class, Soil erosion, forest and agriculture land.

The project proposals were deliberated in the Gram Panchayet meetings which were approved with required amendments.

Based on the experience of the experts working in the area and catchment area characteristics, structures like Check Dam, 
WHS, Stream Bank Stabilisation, Renovation of Farm Ponds, Plantation etc. were provided.

\section{Hydrological modelling}

The relevant hydrological parameters were used for delineation of micro- watersheds as per the existing drainage system. The works/ activities under drainage line treatment are proposed as per stream orders and site condition, stream flow, stream width and length, stream diversions, run- off and topography in consultation with WC. The maps are produced by developing different layers using GIS technology

\section{Results and Discussion}

\section{Preparation of action plan}

Based on the need and problems in watershed area; a draft action plan was prepared and placed before the concerned watershed development committee for approval of the Watershed Committees. After detailed deliberation and incorporation of relevant suggestions into the plan, the action plan was approved in the meeting of Gram Panchayat.

\section{Submission of action plan and approval}

The action plan has been submitted to the State level Agency for scrutiny and technical moderation. After that the action plan has been approved and termed as Detailed Project Report (DPR)

Some initiative has been taken for betterment of the project like as

Geo tagging - An initiative has been taken to record the field observation of all the assets created under Chapramari Watershed through a mobile app namely BHUBAN-DRISHTI as a measures of transparency, accountability, and citizens charter.

Convergence - Some works are advised to be taken with convergence mode with MGNREGA or other Government agency for better management.

PFMS (Public Fund Monitoring system) - To maintain and update the accounting part like DBTL etc of watershed.

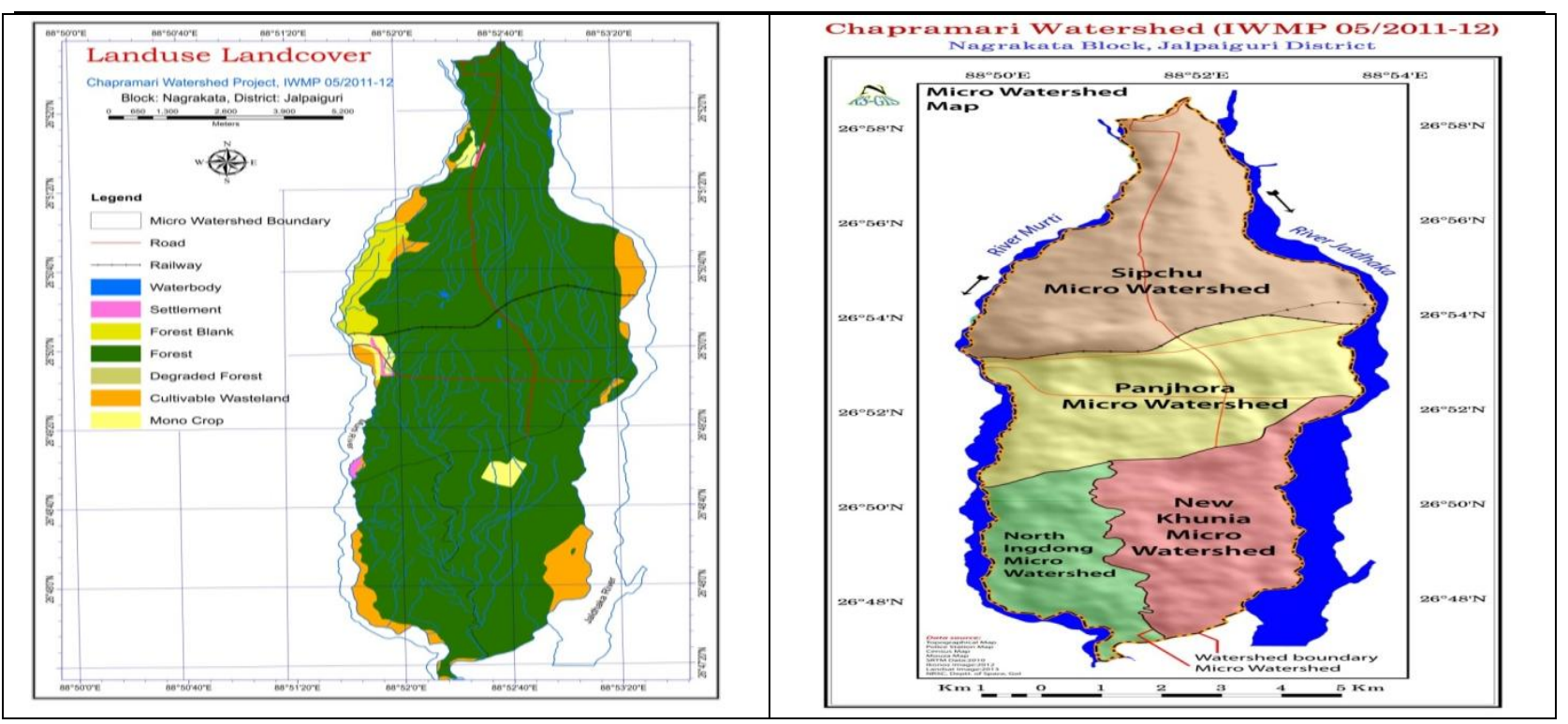



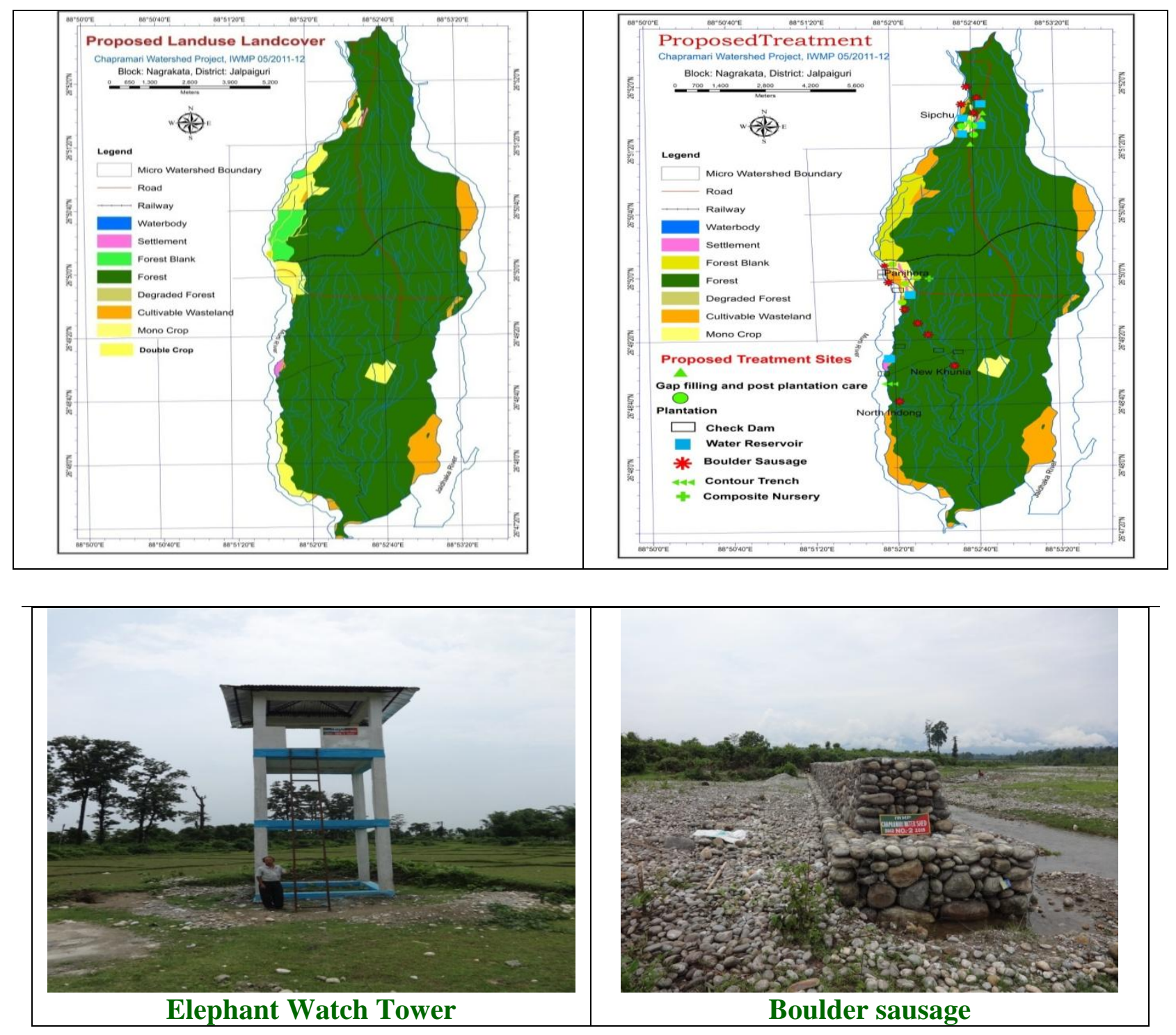

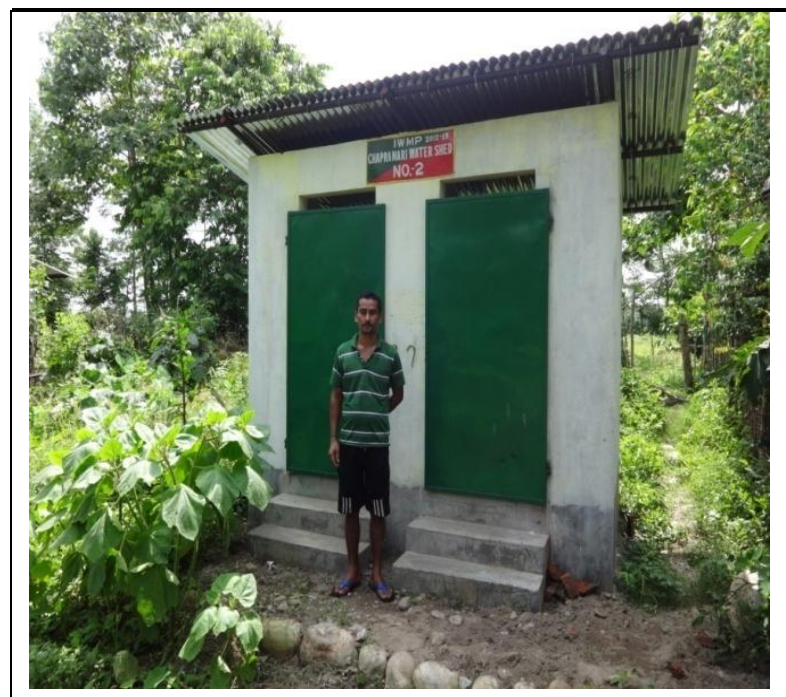

Public sanitation

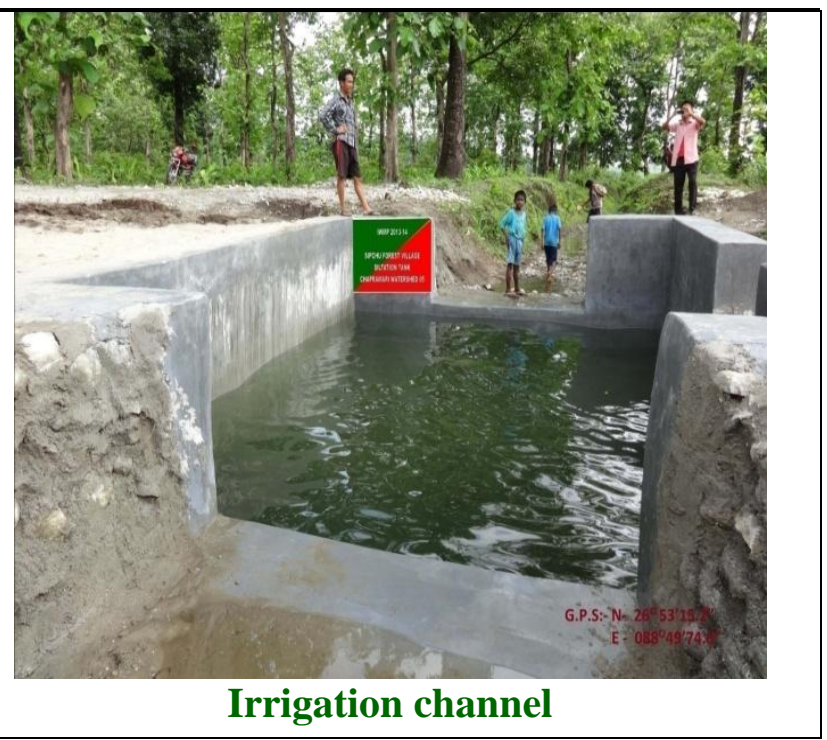


Table.1 Project primary data

\begin{tabular}{|c|c|c|c|c|c|c|}
\hline SI.No & \multicolumn{2}{|c|}{ Particulars } & \multicolumn{4}{|c|}{ Details } \\
\hline 1 & \multicolumn{2}{|c|}{ Project Name } & \multicolumn{4}{|c|}{ CHAPRAMARI WATRSHED } \\
\hline 2 & \multicolumn{2}{|l|}{ Project Code } & \multicolumn{4}{|c|}{ IWMP/ 05 /20 11-12 } \\
\hline 3 & \multicolumn{2}{|l|}{ State } & \multicolumn{4}{|c|}{ WEST BENGAL } \\
\hline 4 & \multicolumn{2}{|l|}{ District } & \multicolumn{4}{|l|}{ JALPAIGURI } \\
\hline 5 & \multicolumn{2}{|l|}{ CD Block } & \multicolumn{4}{|l|}{ NAGRAKATA } \\
\hline 6 & \multicolumn{2}{|l|}{ Gram Panchayat } & \multicolumn{4}{|l|}{ SULKAPARA } \\
\hline \multirow[t]{3}{*}{7} & \multicolumn{6}{|l|}{ Mouza (Village) } \\
\hline & Block & G P & Nam & & Jl.No & $\begin{array}{l}\text { Village } \\
\text { Code }\end{array}$ \\
\hline & NAGRAKATA & SULKAPARA & Upper Tendu Fc & est (M) & $1(\mathrm{M})$ & 00089700 \\
\hline \multirow[t]{5}{*}{8} & \multirow{5}{*}{\multicolumn{2}{|c|}{ Watershed Committee * }} & \multicolumn{2}{|l|}{ Name } & Regd. No. & Remarks \\
\hline & & & \multicolumn{2}{|l|}{ 1) SIPCHU } & S/2L/15373 & \\
\hline & & & \multicolumn{2}{|c|}{ 2) PANJHORA } & $\mathrm{S} / 2 \mathrm{~L} / 15372$ & \\
\hline & & & \multicolumn{2}{|c|}{ 3) NEW KHUNIA } & S/2L/16927 & \\
\hline & & & \multicolumn{2}{|c|}{ 4) NORTH INDONG } & $\mathrm{S} / 2 \mathrm{~L} / 15378$ & \\
\hline \multirow[t]{6}{*}{9} & \multicolumn{2}{|c|}{ Micro watershed Description } & & & & \\
\hline & $\begin{array}{c}\text { Micro } \\
\text { watershed }\end{array}$ & Block & GP & $\begin{array}{l}\text { Name of } \\
\text { Mouza }\end{array}$ & Jl.no & Vill Code \\
\hline & SIPCHU & NAGRAKATA & SULKAPARA & $\begin{array}{c}\text { UPPER } \\
\text { TENDU } \\
\text { (M) }\end{array}$ & $1(\mathrm{M})$ & 00089700 \\
\hline & PANJHORA & NAGRAKATA & SULKAPARA & $\begin{array}{c}\text { UPPER } \\
\text { TENDU } \\
\text { (M) }\end{array}$ & $1(\mathrm{M})$ & 00089700 \\
\hline & NEW KHUNIA & NAGRAKATA & SULKAPARA & $\begin{array}{c}\text { UPPER } \\
\text { TENDU } \\
\text { (M) }\end{array}$ & 1(M) & 00089700 \\
\hline & $\begin{array}{l}\text { NORTH } \\
\text { INDONG }\end{array}$ & NAGRAKATA & SULKAPARA & $\begin{array}{c}\text { UPPER } \\
\text { TENDU } \\
\text { (M) }\end{array}$ & $1(\mathrm{M})$ & 00089700 \\
\hline 10 & \multicolumn{2}{|c|}{ Project Cost (Rs in Lakh) } & & & 516.00 & \\
\hline
\end{tabular}


Table.2 Socio economic profile of the project area

\begin{tabular}{|c|c|c|}
\hline Sl.No & Particulars & Details \\
\hline 1 & Name of Project Implementing Agency (PIA) & $\begin{array}{l}\text { D.F.O Soil conservation Division, } \\
\text { Hakimpara, Jalpaiguri }\end{array}$ \\
\hline 2 & Nature of PIA (a) VO b) Panchayat c) Others) & $\begin{array}{l}\text { Other: - Govt. Of West Bengal } \\
\text { Department of Forest Soil } \\
\text { conservation Division Jalpaiguri }\end{array}$ \\
\hline 3 & Total Population in the Watershed ${ }^{1}$ & 1560 \\
\hline 4 & Total Number of households & 327 \\
\hline 5 & $\begin{array}{l}\text { Total Number of ST households } \\
\text { \{ST Population /(Total Population / Total } \\
\text { Family)\} }\end{array}$ & 118 \\
\hline 6 & $\begin{array}{l}\text { Total Number of SC households } \\
\text { \{SC Population /(Total Population / Total } \\
\text { Family)\} }\end{array}$ & 70 \\
\hline 7 & Total Number of BPL households & 228 \\
\hline 8 & $\begin{array}{l}\text { Total Number of Small Farmer household }{ }^{2} \\
\text { (appox) } \\
\text { (may be Computed from Agri Census 2005-06 and } \\
\text { table 3.14) }\end{array}$ & 99 \\
\hline 9 & $\begin{array}{l}\text { Total number of Marginal farmer household } \\
\text { (appox) (may be Computed from Agri Census } \\
\text { 2005-06 and table 3.14) }\end{array}$ & 190 \\
\hline 10 & $\begin{array}{l}\text { Total number of landless and asset less households } \\
(B P L+\text { Landless families })\end{array}$ & 17 \\
\hline 11 & Number of person days of seasonal migration & 16386 \\
\hline
\end{tabular}


Table.3 Year wise action plan (Physical- only major)

\begin{tabular}{|c|c|c|c|c|c|c|c|c|}
\hline Sl.no & Particulars & Ref table of DPR Format & $2013-14$ & $2014-15$ & $2015-16$ & $2016-17$ & $2017-18$ & Total \\
\hline 1 & \multicolumn{8}{|c|}{ Watershed Development works } \\
\hline 1.3 .6 & $\begin{array}{l}\text { Others (nos) C.C Drain for } \\
\text { irrigation channel (cum) }\end{array}$ & Table 10.3 & 0.00 & 872.83 & 872.83 & 872.83 & & \\
\hline 1.3 .7 & $\begin{array}{l}\text { R.C.C Box culvert/slab } \\
\text { culvert/hume pipe culvert }\end{array}$ & Table 10.3 & 0.00 & 1 no. & 2 no. & 2 no. & 0.00 & 5 nos. \\
\hline 1.3 .8 & $\begin{array}{l}\text { Irrigation Chanel/jhampi } \\
\text { (RMT) }\end{array}$ & Table 10.3 & 2970 & 0.00 & 5160 & 0.00 & 0.00 & 8130.00 \\
\hline 1.3 .9 & $\begin{array}{l}\text { Check dam for diversities } \\
\text { channel (cum) }\end{array}$ & Table 10.3 & 45.54 & 0.00 & 0.00 & 0.00 & 0.00 & 45.54 \\
\hline 1.3 .10 & Boulder sausage works & Table 10.3 & 1266.58 & 978.1525 & 978.1525 & 978.1525 & 978.1525 & 5179.19 \\
\hline 1.3.11 & Retaining wall (cum) & Table 10.3 & 19.80 & 422.24 & 443.84 & 0.00 & 0.00 & 885.88 \\
\hline 1.3 .12 & $\begin{array}{l}\text { C.C water tank with PVC } \\
\text { pipe supply }\end{array}$ & Table 10.3 & 2 nos. & 0.00 & 0.00 & 0.00 & 0.00 & 2 nos. \\
\hline 1.4 & \multicolumn{8}{|c|}{ Water harvesting structure (New) } \\
\hline \multirow[t]{2}{*}{1.4 .1} & Farm Pond (no,) & & 1 & 0.00 & 0.00 & 0.00 & 0.00 & 1 \\
\hline & (Cum) & Table 10.3 & 1083.90 & 0.00 & 0.00 & 0.00 & 0.00 & 1083.90 \\
\hline \multirow[t]{2}{*}{1.4 .4} & Percolation Tank (no.) & Table 10.3 & 1 & 0.00 & 0.00 & 0.00 & 0.00 & 1 nos. \\
\hline & (Cum) & Table 10.3 & 52.27 & 0.00 & 0.00 & 0.00 & 0.00 & 52.27 \\
\hline 1.5 & \multicolumn{8}{|c|}{ Water harvesting structure (renovated) } \\
\hline \multirow{2}{*}{1.5 .1} & Farm Pond (no,) & Table 10.3 & 0.00 & 0.00 & 443.288 & 0.00 & 0.00 & 443.288 \\
\hline & (cum) & Table 10.3 & 0.00 & 0.00 & 0.00 & 0.00 & 0.00 & 0.00 \\
\hline 2.0 & \multicolumn{8}{|l|}{ Entry Point Activities } \\
\hline 2.1 .1 & No. of EPA activities & Table 5.2 & 29 & 0.00 & 0.00 & 0.00 & 0.00 & 29 \\
\hline 2.1 .2 & $\begin{array}{l}\text { No. of water extracting } \\
\text { structures created }\end{array}$ & Table 5.2 & 1 & 0.00 & 0.00 & 0.00 & 0.00 & 1 \\
\hline 3.0 & \multicolumn{8}{|l|}{ Institution \& capacity Building } \\
\hline 3.1 & $\begin{array}{l}\text { SGH Formation (Newly } \\
\text { created) }\end{array}$ & $\begin{array}{l}\text { Table } 3.17 \& \text { Table } 5.6 .1 \\
\text { (new) }\end{array}$ & 0.00 & 11 & 40 & 40 & 40 & 131 \\
\hline 3.2 .1 & No. of SHGs to be assisted & Table 5.6.1 (new) & 0.00 & 11 & 40 & 40 & 40 & 131 \\
\hline 3.2 .2 & Total No, of SHG members & Table 5.6.1 (new) & 0.00 & 110 & 400 & 400 & 400 & 1310 \\
\hline 3.4 & \multicolumn{8}{|l|}{ Formation of Federation } \\
\hline 3.4 .1 & No. of Federation & Table 5.6.1 (new) & 0.00 & 0.00 & 0.00 & 4 & 4 & 8 \\
\hline 3.4 .2 & $\begin{array}{l}\text { Total number of members } \\
\text { of federation }\end{array}$ & Table 5.6.1 (new) & 0.00 & 0.00 & 0.00 & 200 & 200 & 400 \\
\hline 3.5 & \multicolumn{8}{|l|}{ Capacity Building } \\
\hline 3.5 .1 & No. of Training & $\begin{array}{l}\text { New (Chap } 10.3 \text { capacity } \\
\text { building) }\end{array}$ & 0.00 & 20 & 20 & 26 & 20 & 86 \\
\hline 3.5 .2 & No. of Persons to be trained & $\begin{array}{l}\text { New (Chap } 10.3 \text { capacity } \\
\text { building) }\end{array}$ & 0.00 & 1200 & 1200 & 1560 & 1200 & 5160 \\
\hline 5.0 & \multicolumn{8}{|c|}{ Production System \& Microenterprise } \\
\hline 5.3 & Poultry & & & & & & & \\
\hline 5.3.1 & No. of Activities & & 0.00 & 20 & 20 & 25 & 25 & 90 \\
\hline 5.3 .2 & No. of Beneficiaries & & 0.00 & 20 & 20 & 25 & 25 & 90 \\
\hline 5.6 & Goatery & & & & & & & \\
\hline 5.6 .1 & No. of Activities & & 0.00 & 18 & 18 & 20 & 19 & 75 \\
\hline 5.6 .2 & No. of Beneficiaries & & 0.00 & 18 & 18 & 20 & 19 & 75 \\
\hline 5.8 & Piggery & & & & & & & \\
\hline 5.8 .1 & No. Of Activities & & 0.00 & 2.00 & 3 & 3 & 2 & 10 \\
\hline 5.8 .2 & No. Of Beneficiaries & & 0.00 & 2.00 & 3 & 3 & 2 & 10 \\
\hline 5.9 & Horticulture & & & & & & & \\
\hline 5.9 .1 & No. Of Activities & & 0.00 & 3 & 2 & 3 & 2 & 10 \\
\hline 5.9 .2 & No. Of Beneficiaries & & 0.00 & 3 & 2 & 3 & 2 & 10 \\
\hline 5.10 & $\begin{array}{l}\text { Others(vegetable } \\
\text { cultivation) }\end{array}$ & & & & & & & \\
\hline 5.10 .1 & No. Of Activities & & 0.00 & 10 & 10 & 5 & 5 & 30 \\
\hline 5.10 .2 & No. Of Beneficiaries & & 0.00 & 10 & 10 & 5 & 5 & 30 \\
\hline
\end{tabular}


Table.4 Year wise Action plan (Financial- only major) (Rs. In lakh)

\begin{tabular}{|c|c|c|c|c|c|c|c|c|c|}
\hline Sl.no & Particulars & $\begin{array}{l}\text { Ref } \\
\text { Annexure }\end{array}$ & $\begin{array}{l}\text { Unit } \\
\text { Cost }\end{array}$ & 2013-14 & 2014-15 & 2015-16 & 2016-17 & 2017-18 & Total \\
\hline 1 & \multicolumn{9}{|c|}{ Watershed Development works } \\
\hline 1.3 .6 & $\begin{array}{l}\text { Others (nos) C.C } \\
\text { Drain for } \\
\text { irrigation channel } \\
\text { (cum) }\end{array}$ & & 0.00 & 0.00 & 38.359725 & 38.359725 & 38.359725 & 38.359725 & 153.4389 \\
\hline 1.3 .7 & $\begin{array}{l}\text { R.C.C Box } \\
\text { culvert/slab } \\
\text { culvert/hume } \\
\text { pipe culvert }\end{array}$ & & 0.00 & 0.00 & 1.69371 & 1.98643 & 3.38742 & & 7.06756 \\
\hline 1.3 .8 & $\begin{array}{l}\text { Irrigation } \\
\text { Chanel/jhampi }\end{array}$ & & 0.00 & 1.91762 & 0.00 & 2.81177 & 0.00 & 0.00 & 4.72939 \\
\hline 1.3 .9 & $\begin{array}{l}\text { Check dam for } \\
\text { diverting channel } \\
\text { (cum) }\end{array}$ & & 0.00 & 0.71962 & 0.00 & 0.00 & 0.00 & 0.00 & 0.71962 \\
\hline 1.3.10 & $\begin{array}{l}\text { Boulder sausage } \\
\text { works }\end{array}$ & & 0.00 & 13.66812 & 18.747 & 18.747 & 18.746 & 18.746 & 88.65412 \\
\hline 1.3.11 & Retaining wall & & 0.00 & 0.52382 & 12.133365 & 11.542885 & 0.00 & 0.00 & 24.20007 \\
\hline 1.3 .12 & $\begin{array}{l}\text { C.C water tank } \\
\text { with PVC pipe }\end{array}$ & & 0.00 & 0.51949 & 0.00 & 0.00 & 0.00 & 0.00 & 0.51949 \\
\hline 1.4 & \multicolumn{9}{|c|}{ Water harvesting structure (New) } \\
\hline \multirow[t]{2}{*}{1.4 .1} & Farm Pond (no,) & & & 1 & & & & & 1 \\
\hline & & & 0.00 & 1.57337 & 0.00 & 0.00 & 0.00 & 0.00 & 1.57337 \\
\hline 1.4 .4 & \multicolumn{9}{|c|}{ Percolation Tank (no.) } \\
\hline & & & 0.00 & 0.76688 & 0.00 & 0.00 & 0.00 & 0.00 & 0.76688 \\
\hline 1.5 & \multicolumn{9}{|c|}{ Water harvesting structure (renovated) } \\
\hline \multirow[t]{2}{*}{1.5 .1} & Farm Pond (no,) & & 0.00 & 0.00 & 0.00 & 1 nos. & 0.00 & 0.00 & 1 \\
\hline & (cum) & & 0.00 & 0.00 & 0.00 & 3.054 & 0.00 & 0.00 & 3.054 \\
\hline 2.0 & \multicolumn{9}{|c|}{ Entry Point Activities } \\
\hline 2.1 .1 & EPA activities & & & 20.16 & 0.00 & 0.00 & 0.00 & 0.00 & 20.16 \\
\hline 2.1 .2 & $\begin{array}{l}\text { water extracting } \\
\text { structures created }\end{array}$ & & & 0.34845 & 0.00 & 0.00 & 0.00 & 0.00 & 0.34845 \\
\hline 3.0 & \multicolumn{9}{|c|}{ Institution \& capacity Building } \\
\hline 3.2 .1 & $\begin{array}{l}\text { SHGs to be } \\
\text { assisted }\end{array}$ & & $\begin{array}{l}\text { Upto } \\
0.25\end{array}$ & 0.00 & 2.72956 & 9.926 & 9.926 & 9.92644 & 32.508 \\
\hline 3.4 .1 & $\begin{array}{l}\text { Federation } \\
\text { Assisted }\end{array}$ & & $\begin{array}{l}\text { Upto } \\
2.00\end{array}$ & 0.00 & 0.00 & 0.00 & 7.96112 & 5.97088 & 13.932 \\
\hline 5.0 & \multicolumn{9}{|c|}{ Production System \& Microenterprise } \\
\hline 5.3 & Poultry & & & & & & & & \\
\hline 5.3.1 & No. of Activities & & & & 4.8 & 4.8 & 6 & 6 & 21.6 \\
\hline 5.3 .2 & $\begin{array}{l}\text { Beneficiaries } \\
\text { Assisted }\end{array}$ & & & & 20 & 20 & 25 & 25 & 90 \\
\hline 5.6 & Goatery & & & & & & & & \\
\hline 5.6 .1 & No. of Activities & & 0.00 & 0.00 & 4.32 & 4.32 & 4.8 & 4.56 & 18 \\
\hline 5.6 .2 & $\begin{array}{l}\text { Beneficiaries } \\
\text { Assisted }\end{array}$ & & & & 18 & 18 & 20 & 19 & 75 \\
\hline 5.8 & Piggery & & 0.00 & 0.00 & 0.00 & 0.00 & 0.00 & 0.00 & 0.00 \\
\hline 5.8 .1 & No. Of Activities & & & & 0.48 & 0.72 & 0.72 & 0.48 & 2.4 \\
\hline 5.8 .2 & $\begin{array}{l}\text { No. Of } \\
\text { Beneficiaries }\end{array}$ & & & & 2 & 3 & 3 & 2 & 10 \\
\hline 5.9 & Horticulture & & 0.00 & 0.00 & 0.00 & 0.00 & 0.00 & 0.00 & 0.00 \\
\hline 5.9 .1 & No. Of Activities & & & & 0.72 & 0.48 & 0.72 & 0.48 & 2.4 \\
\hline 5.9 .2 & $\begin{array}{l}\text { No. Of } \\
\text { Beneficiaries }\end{array}$ & & & & 3 & 2 & 3 & 2 & 10 \\
\hline 5.10 & \multicolumn{9}{|c|}{ Others(vegetable cultivation) } \\
\hline 5.10 .1 & No. Of Activities & & & & 2.4 & 2.4 & 1.2 & 1.2 & 7.2 \\
\hline 5.10 .2 & $\begin{array}{l}\text { No. Of } \\
\text { Beneficiaries }\end{array}$ & & & & 10 & 10 & 5 & 5 & 30 \\
\hline
\end{tabular}


Table.5 Year wise Action plan in total (Financial) (Rs in Lakh)

\begin{tabular}{|c|c|c|c|c|c|c|c|}
\hline Sl.no & Particulars & $2013-14$ & $2014-15$ & $2015-16$ & 2016-17 & $2017-18$ & Total \\
\hline 1 & Administrative Cost & 10.32 & 10.32 & 10.32 & 10.32 & 10.32 & 51.6 \\
\hline 2 & Monitoring & 1.032 & 1.032 & 1.032 & 1.032 & 1.032 & 5.16 \\
\hline 3 & Evaluation & 0.00 & 1.032 & 0.00 & 2.064 & 2.064 & 5.16 \\
\hline 4 & Watershed Development & 22.28892 & 72.57044 & 76.50181 & 60.49315 & 57.10573 & 288.96 \\
\hline 5 & Entry Point activity & 20.64 & 0.00 & 0.00 & 0.00 & 0.00 & 20.64 \\
\hline 6 & Preparation of DPR & 5.16 & 0.00 & 0.00 & 0.00 & 0.00 & 5.16 \\
\hline 7 & $\begin{array}{l}\text { Capacity and Institution } \\
\text { Building }\end{array}$ & 0.00 & 6.0 & 6.0 & 7.8 & 6.0 & 25.8 \\
\hline 8 & $\begin{array}{l}\text { Livelihood activities for } \\
\text { asset less persons }\end{array}$ & 0.00 & 2.72956 & 9.926 & 17.88712 & 15.89732 & 46.44 \\
\hline 9 & $\begin{array}{l}\text { Production system and } \\
\text { Microenterprise }\end{array}$ & 0.00 & 12.72 & 12.72 & 13.44 & 12.72 & 51.6 \\
\hline \multirow[t]{2}{*}{10} & Consolidation Works & 3.096 & 3.096 & 3.096 & 3.096 & 3.096 & 15.48 \\
\hline & Total & 103.2 & 93.88156 & 100.046 & 110.7911 & 108.0813 & 516 \\
\hline
\end{tabular}

Table.6 Likely Project Outcome (part 1)

\begin{tabular}{|c|c|c|}
\hline 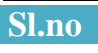 & Particulars & Likely out come \\
\hline 1 & Waste land likely to be converted to productive use (ha) & \\
\hline 1.1 & Total waste land likely to be taken up & 280 \\
\hline 1.4 & Total wasteland likely to be brought under other productive uses & 280 \\
\hline 2 & Cropping intensity & \\
\hline 2.1 & Likely cropping intensity after the project $(\%)$ & $210 \%$ \\
\hline 2.2 & Likely change in cropping intensity (\% increase/ decrease) & 10 \\
\hline 3 & Water Storage Structure/ irrigation & \\
\hline 3.1 & Water Storage Structure (renovated) nos. & 2 \\
\hline 3.2 & Water Storage Structure (created) nos. & 6 \\
\hline 3.3 & Increase in storage capacity of water storage structure (cum) & 443.288 \\
\hline$\overline{3.4}$ & Increase in storage capacity of water storage structure (\%) & 75 \\
\hline 3.5 & Additional water extracting units proposed to be created (nos) & 4 \\
\hline 3.6 & Additional area proposed to be brought under irrigation (ha) & 4 \\
\hline 4 & Likely Beneficiary related outcome & \\
\hline 4.1 & No. of beneficiary household & \\
\hline 4.1 .1 & Small farmers & 99 \\
\hline 4.1 .2 & Marginal farmers & 190 \\
\hline 4.1 .3 & Landless/ asset less & 17 \\
\hline 4.1 .4 & BPL & 228 \\
\hline 4.2 & Expected Flow of benefits (in nos) & 1310 \\
\hline 4.2 .1 & $\mathrm{SC}$ & 70 \\
\hline 4.2 .2 & ST & 118 \\
\hline 4.2 .3 & Minority & nil \\
\hline 4.2 .4 & Others & 139 \\
\hline 5 & Employment likely to be generated & \\
\hline 5.1 & Total number of employment likely to be generated (Person days) & 11184684 \\
\hline
\end{tabular}


Part.2 Expected Flow of benefits (in nos)

\begin{tabular}{|l|l|l|l|l|l|l|l|l|l|l|}
\hline & \multicolumn{4}{|c|}{ SHG } & \multicolumn{4}{|l|}{ Micro } & \multicolumn{3}{|l|}{ Total } \\
\hline 1 & Beneficiaries & M & F & Total & M & F & Total & M & F & Total \\
\hline $\mathbf{2}$ & ST & 34 & 161 & 195 & 35 & 6 & 41 & 69 & 167 & 236 \\
\hline $\mathbf{3}$ & Minority & 121 & 365 & 386 & 73 & 9 & 82 & 194 & 374 & 568 \\
\hline $\mathbf{4}$ & Others & 136 & 491 & 627 & 81 & 11 & 92 & 217 & 502 & 719 \\
\hline & Total & 291 & 1017 & 1208 & 189 & 26 & 215 & 480 & 1043 & 1523 \\
\hline
\end{tabular}

Achievement of the project till date

\begin{tabular}{|c|c|c|c|c|}
\hline $\begin{array}{l}\text { SI } \\
\text { no }\end{array}$ & Component & Unit & Physical Achievement & $\begin{array}{l}\text { Financial } \\
\text { Achievement } \\
\text { (Rs in Lakh) }\end{array}$ \\
\hline \multicolumn{5}{|c|}{ Watershed developmental works } \\
\hline 1 & Boulder Sausage & No & 9 & 21.34 \\
\hline 2 & $\mathrm{CC}$ drain & No & 10 & 11.14 \\
\hline 3 & Irrigation channel & No. & 3 & 1.91762 \\
\hline \multicolumn{5}{|c|}{ Institutional \& Capacity Building } \\
\hline 4 & SHG created & No & $\begin{array}{l}11 \text { (covering } 110 \\
\text { members) }\end{array}$ & $\begin{array}{l}0.25 \text { (as a loan } \\
\text { facility) }\end{array}$ \\
\hline 5 & User Group formation & No & 9 (covering 90 members) & - \\
\hline 6 & Training organized & No & $\begin{array}{l}65 \text { (person trained } 472 \\
\text { nos) }\end{array}$ & - \\
\hline 7 & Providing livelihood assistance & Household & 10 & 2.40 \\
\hline 8 & Exposure visit & No & 4 (person visit 96nos) & - \\
\hline
\end{tabular}

\section{Achievement of the project till date}

Here are the various development works which becomes a success story of the project:

Run off stream has been reduced considerable amount

Soil erosion and nutrient loss has been checked

Due to construction of check dam many artificial water holes created- benefitted to the wild life
Construction of irrigation channel supply the round the year water source to the forest villages for agriculture purpose

Due to increase in the yield in cash crop, cereals, grains- the dependency on forest is reduced.

Socio-economic up-liftment of forest village support the better livelihood with dignity.

\section{Acknowledgement}

The PIA, Chapramari Watershed for providing the data and information. The staffs 
of Watershed Development Team for extending support for field exercise and discussion with the community

\section{References}

Annonymous, 2002. Participatory Resource Appraisal for preparing Watershed action plan, ICAR-Indian Institute of Water management-Research Bulletin.

Annonymous, 2016 Water Management Technologies for Different AgroEcological Condition, ICAR-Indian Institute of Water managementResearch Bulletin.

Bhumela, D.R. 1983. - Small Reservoirs-A programme for improving rainfed agriculture special lecture to the officer trainees in soil conservation, CSWCRTI, Dehradun,

Das, D.C. 1985. - Problem of Soil Erosion and Land degradation in India, National Seminar on Soil Conservation and watershed management, September 1718, New Delhi, 1-24,

Das, Ghanashyam 2009. - Hydrology and Soil Conservation Engineering: Including Watershed Management, PHI Learning,

Das, Madan Mohon, Saikia, Mimi Das 2012. Watershed management, PHI Learning,

Davenport, Thomas E. 2002. - The watershed Project management, CRC Press,

Joshi P. G., 1976. - Soil and water Conservation Technique, Department of Agriculture - Government of Karnataka,

Kumar, Vipin 2011. - Geo-Environmental Study for Watershed Development Planning, A case study of Somb drainage Basin, LAP-Lambert Academic
Publishing,

Lal, Rattan 1999. - Integrated Watershed Management in the Global Eco system, CRC Press,

Lyon, John G. 2002. - GIS for Water Resource and Watershed Management, CRC Press,

Murty, J.V.S. 2012. - Watershed Management, New Age International (P) ltd.,

Prabhu, S and Manikandan, K. 2013. - Indian Forestry- A breakthrough approach to Forest Service, $6^{\text {th }}$ edition, Jain Bros

Rai, Raveendra Kumar 2010. - Hydrological Modeling of Small Watershed, LAPLambert Academic Publishing,

Rao, M.S.V. Rama 1957. - Standardisation of Soil Conservation Practices, Journal of Soil and Water Conservation, India, 6(1):34-38,

Rao, M.S.V. Rama 1974. - Soil Conservation in India, ICAR Research, New Delhi,

Shanmugam C. R. 1977. - Technical handbook on Soil Conservation, Department of Agriculture, Government of Tamil Nadu

Singh, Gurmel, Venkataraman, C, Sastry, G, Joshi, B.P. 2017. - Manual of Soil \& Water Conservation practices, Reprint, Oxford and IBH Publishing CO. Pvt. Ltd.,

The PIA, Chapramari watershed and the Project Manager, WCDC, Jalpaiguri 2012. - Detailed Project Report of Chapramari Watershed, $M / S$ - RS-GIS, Kolkata

Website - PMKSY, IWMP, WBSWDA-West Bengal, ICAR-CSWCRTI, Dehradun.

\section{How to cite this article:}

Mrinal Kanti Roy. 2018. Participatory Rural Appraisals (PRA) for Watershed Management (A Case Study of Chapramari Watershed). Int.J.Curr.Microbiol.App.Sci. 7(01): 1547-1559. doi: https://doi.org/10.20546/ijcmas.2018.701.188 